\title{
Fe-doped $\mathrm{ZnO}$ nanoparticle toxicity: assessment by a
}

\section{new generation of full-particle all-atom}

\section{nanodescriptors}

Jaanus Burk ${ }^{\mathrm{a}}$, Lauri Sikk ${ }^{\mathrm{a}}$, Peeter Burk ${ }^{\mathrm{a}}$, Bella B. Manshian ${ }^{\mathrm{c}, \mathrm{d}}$, Stefaan J. Soenen ${ }^{\mathrm{c}, \mathrm{d}}$, Tarmo Tamm ${ }^{\mathrm{b}}$,

$\underline{\text { Kaido Tämm }}^{\mathrm{a}^{*}}$

${ }^{a}$ Institute of Chemistry, University of Tartu, Ravila 14a, Tartu 50411, Estonia

${ }^{b}$ Institute of Technology, University of Tartu, Nooruse 1, Tartu 50411, Estonia

${ }^{c}$ NanoHealth and Optical Imaging Group, Department of Imaging and Pathology, KU Leuven, B3000 Leuven, Belgium

${ }^{d}$ MoSAIC, KU Leuven, B3000 Leuven, Belgium

*Corresponding author: karu@ut.ee

ABSTRACT. In search of novel tools to combat cancer, nanoparticles (NP) have attracted a lot of attention. Recently, the controlled dissolution of cancer-cell-killing metal ions from doped NPs has shown promise, but fine tuning of dissolution kinetics is required to ensure specificity and minimize undesirable toxic side-effects. Theoretical tools to help in reaching a proper understanding and finally be able to control the dissolution kinetics by NP design have not been available up to now. Here, we present a novel set of true nanodescriptors to analyze the charge distribution, effect of doping and surface coating of whole metal oxide NP structures. The polarizable model of oxygen atoms enables to shed light on the charge distribution on the NP surface, allowing to study in detail the factors influencing the release of metal ions from NPs. The descriptors and their capabilities are demonstrated on a Fe-doped $\mathrm{ZnO}$ nanoparticle system, a system with practical outlook and available experimental data. 
KEYWORDS: Optimized all-atom nanoparticles, Modeling of metal oxide nanoparticles, Nanodescriptors, Nanotoxicity, Nanomodeling;

\section{Introduction}

While nanoparticles (NPs) are already used in medicine as biosensors and drug delivery agents, ${ }^{1,2}$ their biomedical use in, for example, the treatment of cancer, remains challenging. ${ }^{3}$ Aside from many beneficial properties of NPs, their high bioactivity is often associated with toxic side effects, which have been broadly described elsewhere. ${ }^{4,5,6,7,8,9,10,11,12}$

NPs are also used as drug delivery systems due to their improved solubility and pharmacokinetics. ${ }^{13,14}$ One of the studied delivery media is mesoporous silica as it can be modified in multiple ways. ${ }^{15,16}$ Huang et. al. ${ }^{16}$ focused mainly on the shape effects of nano-sized mesoporous silica. They found that the length of the rod determines where the drug will end up and also its clearance rate. For example, short-rods were easily trapped in live. ${ }^{17,18,19}$

(Cyto)toxicity, if delivered in a controlled and selective manner, can be a potent tool for killing cancer cells. This has been demonstrated for example with $\mathrm{ZnO}$ NPs, where dissolution of the NPs and associated release of $\mathrm{Zn}^{2+}$ ions has been shown to specifically affect $\mathrm{p} 53$-mutated cancer cells. ${ }^{20}$ Various studies have demonstrated that the rate of $\mathrm{ZnO}$ dissolution can be affected, for example by coating the $\mathrm{ZnO} \mathrm{NPs}$ with silica or by doping them with $\mathrm{Fe}^{2+}$ ions. ${ }^{21,22}$ As demonstrated, the Fe-doping reduces the toxicity to normal cells and lowers the dissolution rates. Clearly, the line between curing cancer and killing healthy cells is a thin one, therefore, the factors influencing the dissolution kinetics of metal oxide NPs need to be well understood and accurately tunable in order to make this approach valid for real-world application. 
It would be most beneficial, if theoretical analysis could provide the aforementioned understanding, and to allow precise tuning of the dissolution/toxicity of doped metal oxide NPs. First and foremost, atomistic description of large sections of NPs is required to handle doping, and numeric energy/interaction description is required to assess activity - potency for dissolution of surface atoms/ions. Unfortunately, most of the published nano-QSARs contain descriptors that are inherently incapable of such subtlety in NP description.

In 2010, Riviere et.al. ${ }^{23}$ proposed biological surface adsorption indices (BSAI descriptors) to characterize the interactions between chemical groups on the NP surfaces and the amino-acid residues of the proteins by quantifying the competitive adsorption of a set of small molecule probes onto the nanoparticles. From there on, several experimental and computational studies have been carried out for modeling the NP surface interactions in biological systems. ${ }^{24,25,26,27,28}$

While theoretical descriptors for small organic molecules can be readily calculated with various levels of theory to represent most molecular features, the size of NPs (not to mention the scaling with radius) is the obvious limiting factor. Therefore, all approaches have had to choose between handling full NPs at simpler levels of theory or consider just a small fraction of the NPs and use quantum chemical methods. In 2011 Puzyn et al. ${ }^{29}$ studied the cytotoxicity of 17 metal oxide NPs to the bacteria Escherichia coli, making a presumption that the toxicity is independent of the size of the NPs. A correlation was established between the cytotoxicity and the heat of formation for metal cation. Jagiello et al. ${ }^{30}$ has applied size-dependent electronic properties as descriptors for nano-QSAR. However, these parameters were extrapolated from molecular representation and had no relation to the actual size of the whole NP, not to mention the surface structure. 
Toropov et al..$^{31}$ used "optimal" descriptors - something considered as intermediates between classical and nanodescriptors, which were derived from both the structure of NPs (represented as SMILES codes) as well as experimental data related to the substance and the experimental conditions. Other attempts making use of the SMILES codes in NP modeling ${ }^{32,33,34}$ have been made. As NPs are often coated to reduce agglomeration or to promote transport into cells, studies of NPs coated with organic molecules or metal atoms have been carried out. QSAR ${ }^{35}$ representations of those particles have used the presumption that all the properties of NPs depend solely on the structure of the coating, ignoring the underlying NP. It appears that the principal feature of nanomaterials - the high concentration of atoms with high potential energy - has not really been adequately described in any of these studies.

\section{Results and Discussion}

\section{Background of true nanodescriptors}

Previously, we have reported a set of full particle nanodescriptors, ${ }^{36}$ calculated directly and solely from the atomic structure of the NPs, not requiring external information apart from the crystal structure of the respective bulk materials. These nanodescriptors were based on a forcefield calculation of the potential energies of whole NPs and the core and shell layers, and have been proven effective for modeling, grouping and read-across of metal oxide NPs properties and biological activities. ${ }^{22}$ While these first true nanodescriptors were a step forward, they still have shortcomings from the theoretical perspective which also affected in their potential applications.

Firstly, the core and shell region definition ( $1 \mathrm{~nm}$ from surface) was rather arbitrary and not entirely adequate for the development of high precision descriptors allowing to distinguish the energies between the surface and non-surface atoms. Secondly, the atomic surface structure was 
still carved out of bulk material, again not providing enough accuracy at the atomic layer level. The present study takes the approach of whole-particle nanodescriptors a step further by specifiying an approach for the NP-specific definition of the shell and core area of the NPs, as well as surface structure optimization.

The previous set of nanodescriptors ${ }^{36}$ was based on atom counts, potential energies, and coordination numbers. NP structures were generated by replicating the unit cells of the thermodynamically most stable crystal structures and then deleting atoms outside the set radius of NPs. Electroneutrality was achieved by adding an appropriate number of oxygen or metal atoms onto the surface. The drawback of this approach was that the lattice parameters of metal oxides were not taken into account for the added atoms. Thus, two atoms could have ended up too close to each other, or even overlap. This, in turn, can adversely affect the calculation of potential energy descriptors, particularly for the smallest sized NPs. In the current approach, the necessary amount of oxygen or metal atoms (calculated from the number of metal and oxygen atoms and their respective charges), were removed from the surface of NPs to achieve electroneutrality. Such an approach mitigates the possibility of two neighboring atoms having too short or long bond length or overlap due to random placement of new atoms.

Secondly, Lennard-Jones potential ${ }^{37}$ with polarizable oxygen atoms was used for the calculation of energies instead of the Buckingham potential. ${ }^{38}$ Main reasons for changing the potential were: a) the possibility of using the same parametrization for multiple metal oxides, ${ }^{39}$ b) employing polarizable potential to include descriptors based on oxygen polarization and possible extension of this methodology to include coating molecules, as the Lennard-Jones potentials are available for a large number of atoms and atom pairs. 
The NP structures were optimized using the Polak-Ribiere version ${ }^{40}$ of the conjugate gradient algorithm. This is an important advantage over bulk crystal structure-based geometry, as the differences in bond length in core and shell area of the NP are now properly represented. All the above-mentioned modifications enable modeling of more complex (e.g. coated, doped) NPs, and also to develop new nanodescriptors based on atom polarization.

\section{Derivation of nanodescriptors}

In the present approach, the generation of NP structure for computational modeling has been improved. Two novel classes of nanodescriptors are introduced based on (i) the polarized oxygen atoms, and (ii) the force vectors of all atoms. To distinguish between released $\mathrm{Zn}^{2+}$ ions and the ions embodied in the structure of NP, henceforth the latter are called $\mathrm{Zn}$ and $\mathrm{O}$ atoms for the sake of clarity. The parametrization of these properties is crucial as they specifically describe the potential energy (stability, activity) of the atoms on the surface of NPs. The polarization of oxygen atoms is calculated using the adiabatic core-shell method where a shell oxygen atom has 1.5 times the charge of the oxygens in the non-polarized model and the core oxygen has -0.5 of the non-polarized oxygen charge. ${ }^{37}$ The pairwise dipole moment is calculated by defining the distance between the corresponding core-shell oxygen atoms and multiplying it by the absolute value of the sum of the corresponding charges. Force vector magnitude descriptors were calculated at the last step of the optimization by taking into account the average values of atom 
types and NP regions. In addition, the force vector was divided into normal and tangent vector components, based on the coordinates of the atom and the center of the NP (Figure 1).

Radial functions of all calculated properties, such as potential energies, coordination numbers, oxygen dipole moments, force vectors and its components are all dependent on the atom's distance from the NP center. Examples of calculated properties for $\mathrm{ZnO} \mathrm{NP}$ at different shell sizes with radius $82.5 \AA$ are depicted in Figure 2. While the current approach is directly applicable to other metal oxides and doping elements, ZnO NPs with and without Fe doping have been considered here as a (model)system which has both available experimental data and potential practical outlook in cancer treatment.

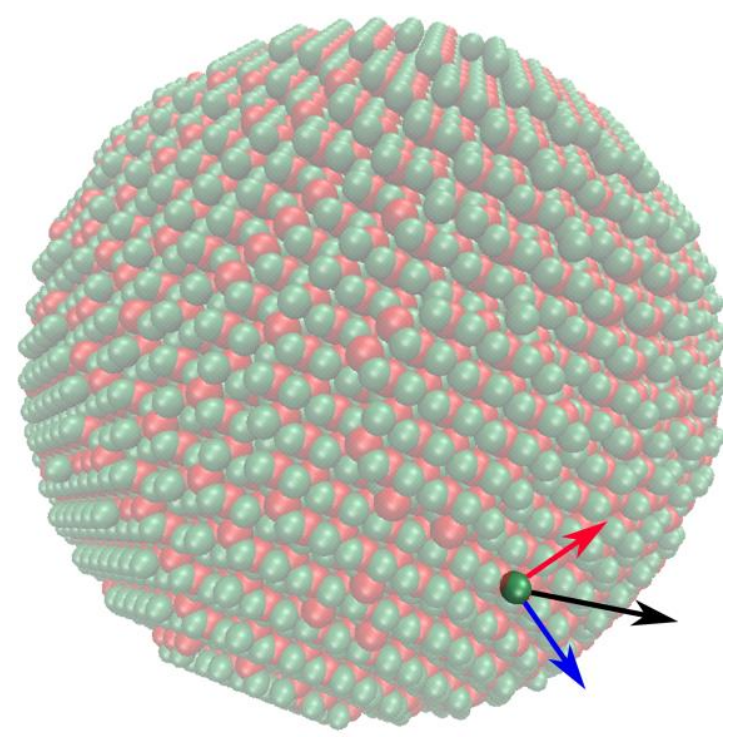

Figure 1. Calculated force vector of the atom (black), force vector normal component (blue) and force vector tangent component (red). 

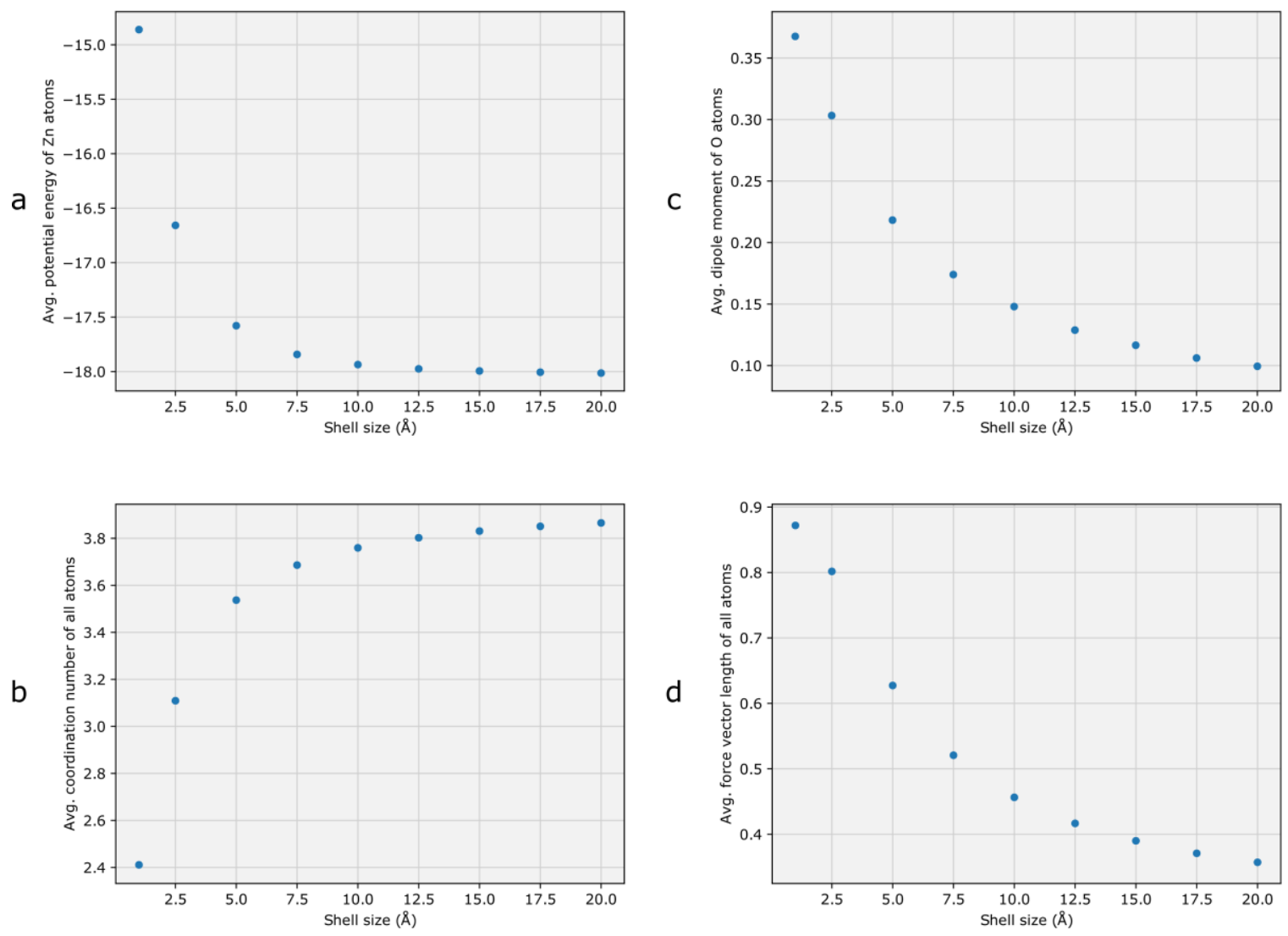

Figure 2. Surface region parameter dependence on the size of surface region a) Potential energy b) Coordination number c) Oxygen dipole moment d) Force vector.

Such a relation is obvious as nearly perfect (bulk) crystal structure occurs in the center of the NP, and as we approach the surface, deviations from the ideal crystal structure become more and more pronounced. This is most notable in case of coordination numbers, as these continuously decrease from 4 in the center to 2.5 on the surface.

While the deviations from the ideal crystal structure start at the NP surface, they progress differently for each property and each chemical composition of the NP. Therefore, it is necessary to take into account the NP-specific thickness of surface region individually for each calculated property and descriptor. The method for defining the NP surface layer thickness is based on the radial functions of the calculated properties. Descriptors based on the potential energies, force 
vector lengths, coordination number and dipole moments of oxygen atoms were all calculated by using different values of shell thickness (from $1 \AA$ up to $20 \AA$ with $2.5 \AA$ step). The calculated descriptor values were plotted against the thickness of shell region (Figure 2). The optimum location for the border of core and shell region is the point of maximum curvature where the core of the NP can be considered to be similar to the bulk metal oxide and the shell region encompasses structural variances due to defects in the lattice structure. To locate the borders of core and shell regions, the Kneedle algorithm ${ }^{41}$ was used for obtaining the curvature data. As it can be seen from Figure $2 \mathrm{a}$ and $2 \mathrm{~b}$, respectively, the potential energy and coordination number descriptors are converging rapidly towards the respective descriptor values of NP core region as the size of the shell region is increased. This is due to the relative interaction distance of the Lennard-Jones potential. On the other hand, dipole moment and length of force vector descriptors decay much slower (Figure 2c, d), which can be attributed to an effect where defects on the surface of NP induce polarization of oxygen atoms toward the centre of NP, which in turn cause the polarization of oxygens deeper in NP. To evaluate the optimum thickness of the surface layer for spherical ZnO NPs, we performed calculations with the following radii: 40; 40,$5 ; 41 ; 41,5 ; 47 ; 50 ; 51,5 ; 53 ; 57 ; 64 ; 69,5 ; 72,5 ; 77 ; 82,5 ; 101 ; 125 ; 150 ; 175$ and $200 \AA . \mathrm{We}$ found that the optimal thickness of shell layer was $5 \AA$ for the potential energy, the force vector length, and the coordination number related descriptors. For calculation of dipole moment descriptors, the respective value was $7.5 \AA$. These values are dependent on the lattice parameters of the metal oxides and the Lennard-Jones potential, and therefore, must be calculated individually for each type of metal oxide. Importantly, the optimal shell layer thickness only depends on the chemical identity (lattice parameters) and not on the radii of NPs. 
To study the dependence of descriptor values on NP size, we calculated all descriptors for the aforementioned $\mathrm{ZnO}$ particles. A total number of 86 nanodescriptors were calculated and the respective numerical values can be found in supporting information (SI). For illustration, plots of three calculated descriptor values as a function of the radius of the NP are shown in Figure 3.

As the size of the NP increases, the lattice energy values (Figure 3a) converge toward those of the bulk metal oxide, which in case of $\mathrm{ZnO}$ is $-38 \mathrm{eV}$. Similarly, the coordination number of $\mathrm{Zn}$ atoms in the NP (Figure 3b) approaches 4 (the value for bulk $\mathrm{ZnO}$ ), and respectively, the average force vector surface normal component approaches 0 (Figure 3c). These different relationships are caused by the spherical shape of the NP (the volume of a sphere scales faster than the surface area of the sphere). 

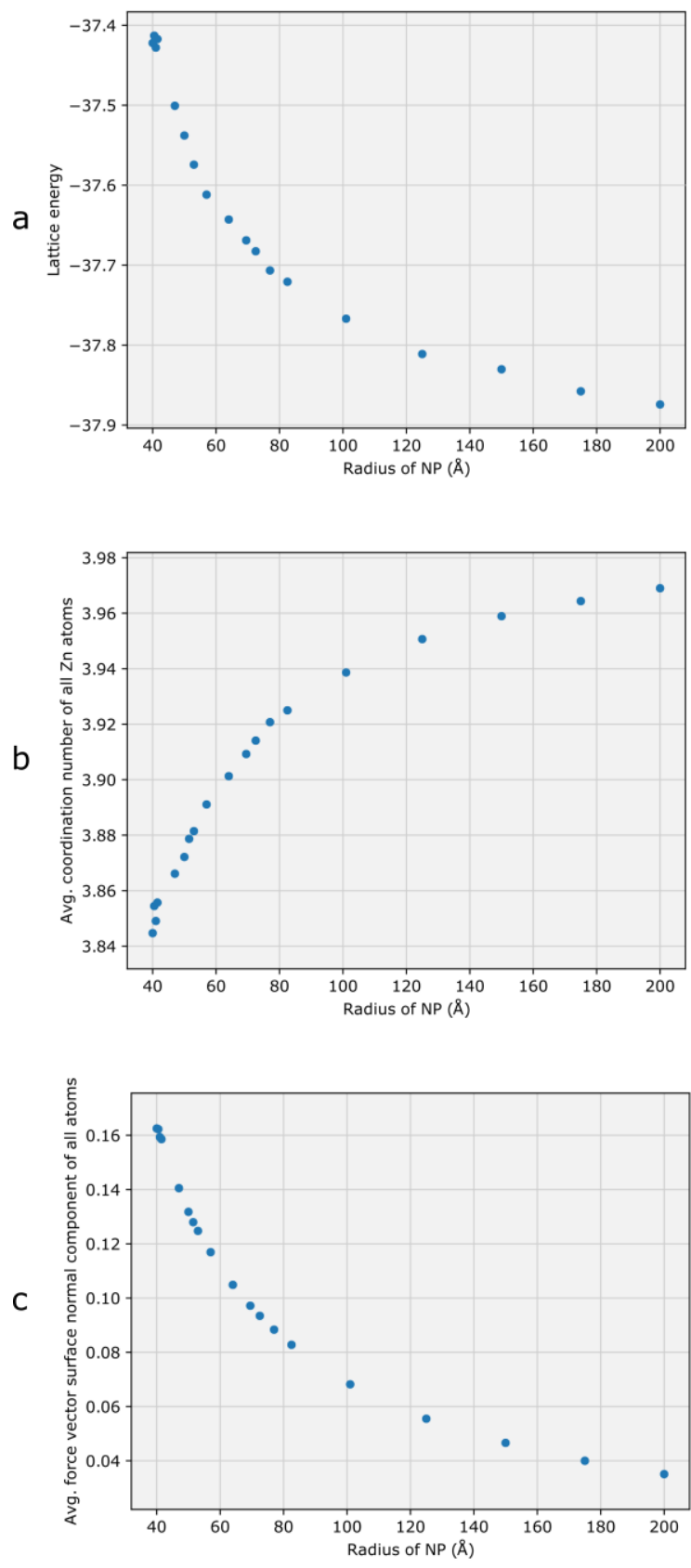

Figure 3. $\mathrm{ZnO}$ nanodescriptor values as a function of NP radius: a) The lattice energy of NP; $\mathrm{b}$ ) The average coordination number of all Zn atoms; c) The average force vector surface normal component of all atoms.

As Fe-doping has been suggested for the ZnO NPs targeting cancer treatment, we calculated the descriptors for $\mathrm{Fe}(\mathrm{II})$ doped $\mathrm{ZnO}$ NPs (Fe\%: 1; 2; 4; 6; 8; 10, NP radius: $69.5 \AA$ ). ${ }^{22}$ The doped NP structures were generated by replacing the required number of $\mathrm{Zn}$ atoms in an NP randomly 
with Fe atoms to achieve the necessary doping level. This simple approach is possible due to the fact that $\mathrm{Zn}$ and $\mathrm{Fe}$ have the same oxidation state, and adding Fe atoms did not cause a change in the overall charge of the NP. In case of different oxidation states or different lattice structure, special care must be taken for constructing doped NPs. Plots of selected descriptor values $v s$. the Fe doping percentage is presented in Figure 4.

As seen in Figure $4 \mathrm{a}$ and $4 \mathrm{~b}$, potential energy descriptors exhibit nearly linear correlation with the Fe content in the NP. This is due to the difference in lattice parameters and the interatomic potential of the respective oxides. Fe-O bond length in wurtzite structure is $2.167 \AA^{42}$ while the $\mathrm{Zn}-\mathrm{O}$ bond length in wurtzite structure is $1.98 \AA{ }^{43}$ Therefore, the insertion of Fe atoms in the lattice of $\mathrm{ZnO}$ causes local distortions in the crystal structure and decreases the potential energy - the lattice energy becomes less negative (Figures $4 a$ and $4 b$ ). 

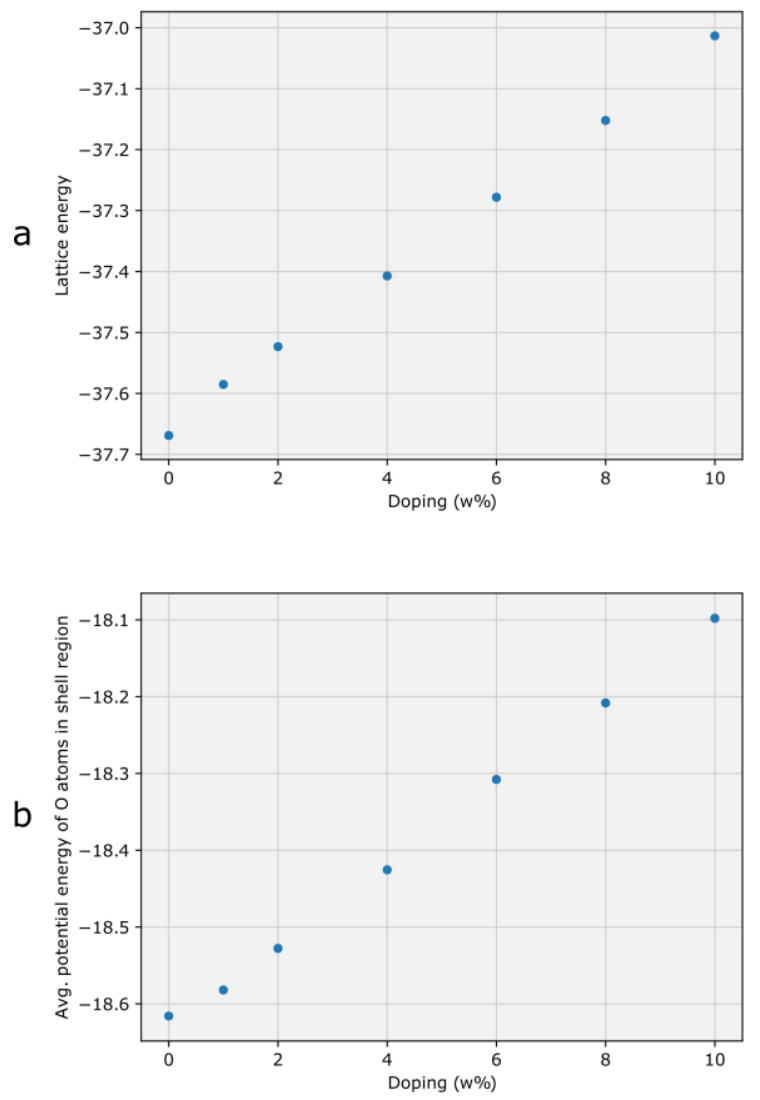

Figure 4. Plot of selected descriptor values vs. the Fe doping percentage; A) Lattice energy of $N P B)$ Average potential energy of oxygen atoms in shell region.

Combining the size and doping dependencies of nanodescriptors, one can see that the size and $\mathrm{Fe}$ content may have a very different effect on their values. In case of lattice energy, the increase of NP radius stabilizes the NP while the increase in Fe content causes the opposite effect.

To study the systematic behavior of lattice energy depending on the size and doping level of NPs, descriptor values were calculated for 19 different sizes and 7 different doping percentages (133 combinations in total) of $\mathrm{ZnO}$ NPs. Figure 5 displays the size and doping level dependence of the lattice energy descriptor. 

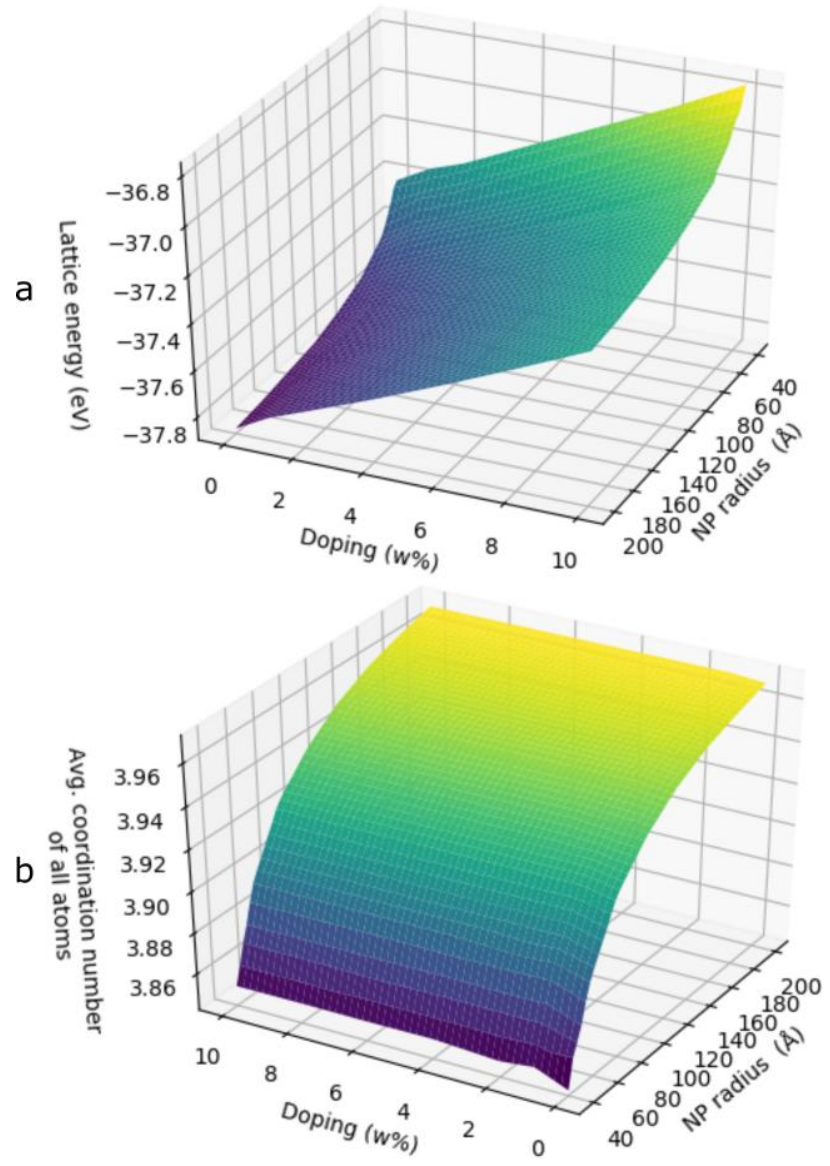

Figure 5. Effect of doping (weight percent) and size of ZnO NPs on the calculated lattice energy and average coordination number of all atoms descriptors.

Figure 5 demonstrates that the destabilizing effect, caused by the increasing number of doping Fe atoms (or overall NP size) is similar for all the calculated NPs. This outcome enables the development of a two-parameter linear model, which can be used to apply the novel nanodescriptors in nano-QSARS without explicitly performing the force-field calculations on particular NPs. The linear model allows the interpolation of descriptor values based on the radius and amount of $\mathrm{Fe}($ weight $\%$ ) in NP:

$$
D=a+b * F e(w \%)+c *\left(\frac{1}{R}\right)
$$

where $\mathrm{D}$ is the value of the predicted descriptor, $\mathrm{a}, \mathrm{b}, \mathrm{c}$ are equation coefficients, $\mathrm{Fe}(\mathrm{w} \%)$ is doping level (weight \%) and $\mathrm{R}$ is the radius of NPs in angstroms. The coefficient values for three 
descriptors: the lattice energy of $N P$, average coordination numbers of all atoms and average length of force vector surface normal component for all Zn atoms are presented in Table 1.

Table 1. Parameters for interpolation of descriptor values based on the size and composition of NP.

\begin{tabular}{|c|c|c|c|c|c|}
\hline Descriptor & b & & c & $\mathbf{R}^{2}$ & $\mathbf{R}^{2} \mathrm{cv}$ \\
\hline 1. Lattice energy of $N P$ & -38.99 & $6.451 \cdot 10^{-2}$ & 22.09 & 0.965 & 0.964 \\
\hline 2. Avg. coord. No. of all atoms & 4.00 & $2.298 \cdot 10^{-4}$ & -6.02 & 0.998 & 0.998 \\
\hline $\begin{array}{l}\text { 3. Avg. length of force vector } \\
\text { surface normal component for } \\
\text { all Zn atoms }\end{array}$ & $2.90 \cdot 10^{-2}$ & $-3.376 \cdot 10^{-3}$ & 11.15 & 0.934 & 0.931 \\
\hline
\end{tabular}

The statistical fit between the calculated and extrapolated descriptor values are very good, $\mathrm{R}^{2}$ varies between 0.934 and 0.998 . Leave one out cross-validation $\left(\mathrm{R}^{2} \mathrm{CV}\right.$, Table 1) was performed for these multilinear equations, which demonstrates the predictive power of the constructed models. All of these descriptors depend on the reciprocal value of the NP radius. This is caused by the spherical shape of the NPs, as the surface area to volume ratio of spheres is also a function of $1 / \mathrm{R}$. The relationship between the NP size and calculated descriptors is in agreement with previously published results. ${ }^{44}$ Size dependent changes of some NP properties occur only in case of small NPs (diameter below $\sim 30 \mathrm{~nm}$ ) and therefore it is crucial to take the size of NP into account when calculating nanodescriptors and using these to predict the properties of other NPs.

\section{Application of nanodescriptors: Fe-doped ZnO NPs and cancer cells}

The developed descriptors were applied in the analysis of the biological effect of doped NPs on the KLN205 and HeLa cell lines. The biological data for different parameters of these cell lines were obtained from a study by Manshian et. al. ${ }^{22}$ There, high-content based multiparametric screening had been applied to investigate the biological effects of the Fe-doped ZnO NPs. Here, cells were exposed to different concentrations of the various Fe-doped $\mathrm{ZnO} \mathrm{NPs}$ for $8 \mathrm{hr}$ and 
then stained and analyzed using a previously validated high-content imaging methodology optimized for use in bio-nano interactions studies. ${ }^{45}$ In current study, the following endpoints were selected: (i) occurrence of cell death, (ii) membrane damage and (iii) the induction of reactive oxygen species $(30 \mu \mathrm{g} / \mathrm{ml}, 8 \mathrm{hr}$ exposure). These three parameters were chosen as they represent the proposed toxic mechanism of $\mathrm{ZnO}$ NPs (induction of reactive oxygen species) and measured effect on the vitality of cells (occurrence of cell death and membrane damage). While statistically significant linear correlations between the calculated descriptor values and the measured parameters ( 7 NPs with different doping weight percent) were established, the small number of data-points does not really support focusing on the correlation statistics too much, as is so often the case with NP bioactivity. Therefore, more attention should be paid to the mechanistic relations and descriptor value trends.

As the proposed mechanism of toxicity of Fe doped $\mathrm{ZnO}$ nanoparticles is the release of $\mathrm{Zn}^{2+}$ ions from the surface of a NP, ${ }^{46}$ the amount of available $\mathrm{Zn}$ on the surface can be expected to correlate with the cell death, membrane damage and mitochondrial ROS. Statistical parameters for these one-parameter equations are presented in Table 2. As visible from Table 2, the slope for the equations relating the number of $\mathrm{Zn}$ atoms to biological endpoints is positive, indicating a logical relationship between the descriptor and the measured property - increasing the number of available $\mathrm{Zn}$ atoms on the surface of NPs also increases the toxicity of the NPs. It is interesting to note that the statistical parameters for mitochondrial ROS are slightly higher than those for cell death. One possible explanation is that the generation of reactive oxygen species is the primary outcome from the release of $\mathrm{Zn}^{2+}$ ions from the surface of $\mathrm{NP}$, while cell death is the sum of several factors and mechanisms resulting from the interaction with NPs.

Table 2. Statistical parameters for the correlations of the three endpoints of the two cell lines with the log number of Zn atoms in shell region of NP. 


\begin{tabular}{llllll} 
Cell line & Endpoint (unitless) & Slope & Intercept & $\mathbf{R}^{\mathbf{2}}$ & $\mathbf{R}^{\mathbf{2}} \mathbf{c v}$ \\
\hline HeLa & Cell death & 1.60 & -4.69 & 0.947 & 0.837 \\
HeLa & Membrane damage & 2.44 & -7.55 & 0.969 & 0.919 \\
HeLa & Mitochondrial ROS & 3.81 & -11.79 & 0.977 & 0.962 \\
KLN205 & Cell death & 1.86 & -5.63 & 0.950 & 0.856 \\
KLN205 & Membrane damage & 2.62 & -8.17 & 0.965 & 0.907 \\
KLN205 & Mitochondrial ROS & 4.01 & -12.49 & 0.965 & 0.938 \\
\hline
\end{tabular}

*Respective plots are presented in Supplementary Information, Figure S1.

While the number of $\mathrm{Zn}$ atoms on the surface of the NP was shown to be important to describe the toxic effects of doped NPs, the count itself cannot possibly be the only factor as the larger NPs should have relatively less active surface atoms, also doping may change surface atom activity. Therefore, one also has to take into account the chemical surroundings of the $\mathrm{Zn}$ ions in the NP, e.g. the potential energy of the $\mathrm{Zn}$ atoms. One possible approach to numerically characterize the potential energy in a crystalline material is via lattice-energy. For that end, oneparameter equations for the lattice energy of NP/Surface area of NP versus selected biological endpoints were generated (Table 3). The inclusion of the surface area in the descriptor reduces the role of the size of the NP, and therefore, amplifies the effect of doping with other elements, like Fe in our case.

Table 3. Statistical parameters for the correlations of the three endpoints of the two cell lines with the descriptor lattice energy of NP/Surface area of NP.

\begin{tabular}{llllll} 
Cell line & Endpoint (unitless) & Slope & Intercept & $\mathbf{R}^{\mathbf{2}}$ & $\mathbf{R}^{\mathbf{2} \mathbf{}}$ \\
\hline HeLa & Cell death & 1008 & 2.44 & 0.914 & 0.759 \\
HeLa & Membrane damage & 1552 & 3.34 & 0.951 & 0.868 \\
HeLa & Mitochondrial ROS & 2439 & 5.21 & 0.974 & 0.948 \\
KLN205 & Cell death & 1173 & 2.66 & 0.917 & 0.776 \\
KLN205 & Membrane damage & 1661 & 3.52 & 0.941 & 0.841 \\
KLN205 & Mitochondrial ROS & 2570 & 5.41 & 0.963 & 0.919 \\
\hline
\end{tabular}

*Respective plots are presented in Supplementary Information, Figure S2.

Again, the mitochondrial ROS correlates better with the lattice energy of NP/Surface area of NP than the cell death or the membrane damage. 
If we want to represent both the size of the NP and the chemical nature of the surface of metal oxide NPs as a single stable descriptor, we should take a look at the species that is always there oxygen. Fe doping or not, these are still metal oxides. The biological endpoints were correlated against the descriptor average force vector surface normal component of oxygen atoms in shell region (Table 4), as this descriptor characterizes the stability of oxygen atoms on the surface of NP, somewhat indirectly describing the activity of all atoms on the surface of the NP.

Table 4. Statistical parameters for the correlations of the three endpoints of the two cell lines with the descriptor: Average force vector surface normal component of oxygen atoms in shell region.

\begin{tabular}{llllll} 
Cell line & Endpoint (unitless) & Slope & Intercept & $\mathbf{R}^{2}$ & $\mathbf{R}_{\mathbf{c v}}{ }^{\mathbf{c}}$ \\
\hline HeLa & Cell death & 8.27 & -0.25 & 0.877 & 0.789 \\
HeLa & Membrane damage & 12.08 & -0.66 & 0.821 & 0.740 \\
HeLa & Mitochondrial ROS & 18.92 & -1.07 & 0.836 & 0.746 \\
KLN205 & Cell death & 9.54 & -0.45 & 0.866 & 0.784 \\
KLN205 & Membrane damage & 13.11 & -0.80 & 0.836 & 0.757 \\
KLN205 & Mitochondrial ROS & 20.18 & -1.25 & 0.847 & 0.778 \\
\hline *Respective plots are presented in Supplementary Information, Figure S3.
\end{tabular}

While the statistical parameters for the correlations with Average force vector surface normal component of oxygen atoms in shell region appear slightly lower than those of the descriptors considered above, the parameters are remarkably even, suggesting stable performance. The slightly lower statistical parameters are likely related to the fact that zero doping (pure $\mathrm{ZnO}$ ) particles are included in the dataset.

\section{Interpretation of the model}

The present model looks at the toxicity of released $\mathrm{Zn}^{2+}$, but the model in itself cannot really differentiate between the impact of released $\mathrm{Zn}^{2+}$ ions or that of surface-associated $\mathrm{Zn}$ atoms. This largely stems from the lack of experimental data, where the separate influence of both components is very difficult, if not nearly impossible to study. While some studies replace the 
NP-released $\mathrm{Zn} 2+$ by exposing cells to exogenous $\mathrm{Zn} 2+$ ions, this will not result in the same exposure kinetics nor in the same distribution of $\mathrm{Zn}^{2+}$ ions within the cells (for NP-released $\mathrm{Zn}^{2+}$, there will be local high accumulation of ions in the lysosomal compartments, which is not the case for freely added $\mathrm{Zn}^{2+}$ ions). While in previous work, we found that oxidative stress and cellular toxicity correlated with the level of free $\mathrm{Zn}^{2+}$ in solution, it was not possible to draw any correlations on the level of $\mathrm{Zn}^{2+}$ ions still present at the NP surface at different time points. Without the experimental data to draw any conclusion, no model can be accurately distilled to generate any sound conclusions. At present, toxicity has mainly been associated to free $\mathrm{Zn}^{2+}$ ions in literature and also in our own work. Given the high effect of Fe-doping even at low levels (12\%) on NP toxicity, correlating with high effects on dissolution kinetics, yet having such low levels of Fe-ions and assuming a homogeneous distribution of Fe-ions within the $\mathrm{ZnO}$ crystal structure, we conclude that the difference in level of surface-associated $\mathrm{Zn}^{2+}$ ions for these conditions will be minimal and hereby can only play a minor role in the overall differences in cellular toxicity observed for these NP formulations.

The present model furthermore mainly works through an indirect manner, in which various parameters that are linked to $\mathrm{Zn}^{2+}$ release are taking into account, without looking at direct parameters such as the solubility constant. For cell-free systems the concentration of dissolved ions can be measured as a function of time and the solubility constant can be determined, but this is not straightforward to do in cellular environments. Here, any such values would not be very insightful due to differences between cells in view of the subcellular compartmentalization of the NPs and any associated ions that have been released. This results in local differences in concentrations of ions and NPs within the same cell and between different cells in a single population. Furthermore, these differences in final biological compartmentalization will further 
influence the kinetics and extent of ion release due to differences in local $\mathrm{pH}$, and ionic strength of the microenvironment. Additionally, as zinc is an essential element for cellular homeostasis, zinc distribution is linked to active transport mechanisms, which will affect the solubility constant and will, depending on the local concentrations present, change as a function of time. The maintenance of the zinc homeostasis is achieved generally through two main mechanisms: Zinc transporting proteins and zinc-buffer system. The former contains two large metaltransporter families, zinc transporters (ZnTs) and Zrt-Irt-like proteins (ZIPs) that actively transport zinc among cytosol, discrete intracellular compartments, and extracellular medium. Taking these factors into account, any direct parameters cannot be introduced in the current model, given the lack of experimental data that can be obtained to support these in the relevant model system, being a cellular environment and the use of parameters associated to $\mathrm{Zn}^{2+}$ release is, at present, the best way forward.

For comparative analysis between the current nanodescriptors and previous set, ${ }^{22}$ one-parameter models of descriptor vs biological endpoint were calculated. In case of the log number of $Z n$ atoms in shell region of $N P$, the obtained $R^{2}$ and $R_{c v}^{2}$ values were found to be lower than the respective values for current models (average decrease: $R^{2}=0.007$ and $R_{c v}^{2}=0.015$ ). Decrease of $R^{2}$ and $R_{c v}^{2}$ was significantly higher for the lattice energy of NP/Surface area of NP: average decrease $R^{2}=0.037$ and $R_{c v}^{2}=0.078$. While the raise of statistical parameters for the log number of Zn atoms in shell region of NP is affected by different size of shell region and optimization of the NP, the lattice energy of NP/Surface area of $N P$, is influenced also by the change in interatomic potential. 


\section{Conclusions}

None of the previously published nanodescriptors have been capable of analyzing the activity of metal atoms on the surface of metal oxide NPs in detail enough to be applicable to their release from doped metal oxide NPs of different size. In the present work, a number of important improvements to the previous set of full-particle all-atom theoretical nanodescriptors has been achieved, which for the first time allow theoretical insight into NP characteristics of such detail. Firstly, a methodology for calculating the optimal thickness of the (highly active) surface layer of NPs was described, together with surface atom optimization, which enable to derive new descriptors as well as fine-tune those of the previous set to be more consistent and more generally applicable. Secondly, a set of new nanodescriptors was developed, based on the force vectors of all atoms and the "dipole moments" of oxygen atoms, which allow to distinguish between the regular (bulk) NP atoms and those carrying the special properties of NPs - high surface activity.

To prove the adequacy of these developments, the calculated descriptors were used to model the cell death, membrane damage and mitochondrial ROS of Fe-doped $\mathrm{ZnO}$ nanoparticles for two cancerous cell lines. The new and improved nanodescriptors were shown to correlate well with the endpoints, as well as to allow analysis of the mechanisms governing the endpoints for doped metal oxide NPs of different sizes. While Fe(II) doped ZnO system has been studied here, the nanodescriptors and the overall approach is applicable to any metal oxide NPs with various dopings.

\section{Supporting information available}


Examples of newly calculated descriptors for three NPs $(0 \% \mathrm{Fe}, 6 \% \mathrm{Fe}$ and $10 \% \mathrm{Fe})$, experimental values of biological endpoints (unitless), doping amount and radii for modeled NPs, plots for tables 2-4 and the descriptor matrix for all 133 NPs calculated in this work can be downloaded from www.ut.ee/cc/nanodescriptors

\section{Acknowledgements}

This material is based upon work supported by the EU Commission (MODERN, Contract no. 309314, Horizon 2020 ERC to SJS (ERC StG no. 757398), by the institutional research funding IUT (IUT20-15) of the Estonian Ministry of Education and Research and by the Fonds Voor Wetenschappelijk Onderzoek (FWO-Vlaanderen; KAN to BM and SJS). 


\section{References}

1. S. S. Agasti, S. Rana, M.-H. Park, C. K. Kim, C.-C. You and V. M. Rotello, Adv. Drug Deliver Rev., 2010, 62, 316-328.

2. W. H. De Jong and P. J. A. Borm, Int. J. Nanomed., 2008, 3, 133-149.

3. H. S. Huang and J. F. Hainfeld, Int. J. Nanomed., 2013, 8, 2521-2532.

4. $\quad$ A. Nel, T. Xia, L. Madler and N. Li, Science, 2006, 311, 622-627.

5. M. Horie, H. Kato, K. Fujita, S. Endoh and H. Iwahashi, Chem. Res. Toxicol., 2012, 25, 605-619.

6. T. Xia, M. Kovochich, M. Liong, L. Maedler, B. Gilbert, H. Shi, J. I. Yeh, J. I. Zink and A. E. Nel, Acs Nano, 2008, 2, 2121-2134.

7. A. Manke, L. Wang and Y. Rojanasakul, BioMed Res. Int., 2013, 2013, 942916-942916.

8. A. Kahru and H.-C. Dubourguier, Toxicology, 2010, 269, 105-119.

9. I. Passagne, M. Morille, M. Rousset, I. Pujalte and B. L'Azou, Toxicology, 2012, 299, 112-124.

10. A. Avalos, A. Isabel Haza, D. Mateo and P. Morales, J. Appl. Toxicol., 2014, 34, 413423.

11. M. Misawa and J. Takahashi, Nanomedicine, 2011, 7, 604-614.

12. M. Horie, K. Fujita, H. Kato, S. Endoh, K. Nishio, L. K. Komaba, A. Nakamura, A. Miyauchi, S. Kinugasa, Y. Hagihara, E. Niki, Y. Yoshida and H. Iwahashi, Metallomics, 2012, 4, 350-360.

13. Q. A. Pankhurst, J. Connolly, S. K. Jones and J. Dobson, Journal of Physics D-Applied Physics, 2003, 36, R167-R181.

14. R. Gupta and B. Rai, Nanoscale, 2018, 10, 4940-4951.

15. N. Z. Knezevic and J. O. Durand, Nanoscale, 2015, 7, 2199-2209.

16. X. L. Huang, L. L. Li, T. L. Liu, N. J. Hao, H. Y. Liu, D. Chen and F. Q. Tang, Acs Nano, 2011, 5, 5390-5399.

17. X. T. Pan, L. X. Bai, H. Wang, Q. Y. Wu, H. Y. Wang, S. Liu, B. L. Xu, X. H. Shi and H. Y. Liu, Advanced Materials, 2018, 30.

18. T. X. Liang, L. G. Xiao, C. Liu, K. Gao, H. M. Qin, Y. Cao and X. B. Peng, Organic Electronics, 2016, 29, 127-134.

19. J. Bogdan, J. Plawinska-Czarnak and J. Zarzynska, Nanoscale Research Letters, 2017, 12.

20. M. I. Setyawati, C. Y. Tay and D. T. Leong, Biomaterials, 2013, 34, 10133-10142.

21. S. L. Chia and D. T. Leong, Heliyon, 2016, 2, 18.

22. B. B. Manshian, S. Pokhrel, U. Himmelreich, K. Tamm, L. Sikk, A. Fernandez, R. Rallo, T. Tamm, L. Madler and S. J. Soenen, Advanced Healthcare Materials, 2017, 6, 11.

23. X. R. Xia, N. A. Monteiro-Riviere and J. E. Riviere, Nature Nanotechnology, 2010, 5, 671-675.

24. X. R. Xia, N. A. Monteiro-Riviere, S. Mathur, X. F. Song, L. S. Xiao, S. J. Oldenberg, B. Fadeel and J. E. Riviere, Acs Nano, 2011, 5, 9074-9081.

25. R. Chen, Y. Zhang, F. D. Sahneh, C. M. Scoglio, W. Wohlleben, A. Haase, N. A. Monteiro-Riviere and J. E. Riviere, Acs Nano, 2014, 8, 9446-9456.

26. W. Y. Wang, A. Sedykh, H. N. Sun, L. L. Zhao, D. P. Russo, H. Y. Zhou, B. Yan and H. Zhu, Acs Nano, 2017, 11, 12641-12649. 
27. M. Gonzalez-Durruthy, L. C. Alberic, C. Curti, Z. Naal, D. T. Atique-Sawazaki, J. M. Vazquez-Naya, H. Gonzalez-Diaz and C. R. Munteanu, Journal of Chemical Information and Modeling, 2017, 57, 1029-1044.

28. M. Gonzalez-Durruthy, A. V. Werhli, L. Cornetet, K. S. Machado, H. Gonzalez-Diaz, W. Wasiliesky, C. P. Ruas, M. A. Gelesky and J. M. Monserrat, Rsc Advances, 2016, 6, 58680-58693.

29. T. Puzyn, B. Rasulev, A. Gajewicz, X. Hu, T. P. Dasari, A. Michalkova, H.-M. Hwang, A. Toropov, D. Leszczynska and J. Leszczynski, Nat. Nanotechnol., 2011, 6, 175-178.

30. K. Jagiello, B. Chomicz, A. Avramopoulos, A. Gajewicz, A. Mikolajczyk, P. Bonifassi, M. G. Papadopoulos, J. Leszczynski and T. Puzyn, Structural Chemistry, 2017, 28, 635643.

31. A. P. Toropova, A. A. Toropov, R. Rallo, D. Leszczynska and J. Leszczynski, Ecotox. Environ. Safe., 2015, 112, 39-45.

32. A. A. Toropov, A. P. Toropova, T. Puzyn, E. Benfenati, G. Gini, D. Leszczynska and J. Leszczynski, Chemosphere, 2013, 92, 31-37.

33. T. Puzyn, D. Leszczynska and J. Leszczynski, Small, 2009, 5, 2494-2509.

34. A. A. Toropov, A. P. Toropova, E. Benfenati, G. Gini, T. Puzyn, D. Leszczynska and J. Leszczynski, Chemosphere, 2012, 89, 1098-1102.

35. S. Kar, A. Gajewicz, T. Puzyn and K. Roy, Toxicol. in Vitro, 2014, 28, 600-606.

36. K. Tämm, L. Sikk, J. Burk, R. Rallo, S. Pokhrel, L. Madler, J. J. Scott-Fordsmand, P. Burk and T. Tamm, Nanoscale, 2016, 8, 16243-16250.

37. J. E. Jones, Journal, 1924, 463-477.

38. R. A. Buckingham, P. Roy. Soc. Lond. A Mat., 1938, 168, 264-283.

39. L. F. Zhao, L. C. Liu and H. Sun, Journal of Physical Chemistry C, 2007, 111, 1061010617.

40. E. Polak and G. Ribiere, Journal, 1969, 3, 35-43.

41. V. Satopaa, J. Albrecht, D. Irwin and B. Raghavan, Minneapolis, MN, 2011.

42. M. Boiocchi, F. Caucia, M. Merli, D. Prella and L. Ungaretti, European Journal of Mineralogy, 2001, 13, 871-881.

43. Y. N. Xu and W. Y. Ching, Physical Review B, 1993, 48, 4335-4351.

44. J. L. Ying, T. Zhang and M. Tang, Nanomaterials, 2015, 5, 1620-1637.

45. B. B. Manshian, U. Himmelreich and S. J. Soenen, Chemical Research in Toxicology, 2017, 30, 595-603.

46. T. A. Xia, Y. Zhao, T. Sager, S. George, S. Pokhrel, N. Li, D. Schoenfeld, H. A. Meng, S. J. Lin, X. Wang, M. Y. Wang, Z. X. Ji, J. I. Zink, L. Madler, V. Castranova, S. Lin and A. E. Nel, Acs Nano, 2011, 5, 1223-1235. 